\title{
Anger, depression and anxiety associated with endothelial function in childhood and adolescence
}

\author{
W Osika, 1,2 S M Montgomery, 2,3 F Dangardt,1 P Währborg, ${ }^{1}$ L M Gan, \\ E Tideman, ${ }^{4}$ P Friberg ${ }^{1}$
}

'Department of Metabolism and Cardiovascular Research/ Clinical Physiology,

Sahlgrenska University Hospital, Göteborg, Sweden ${ }^{2}$ Clinical Epidemiology Unit, Department of Medicine, Karolinska Hospital, Karolinska Institutet, Stockholm, Sweden ${ }^{3}$ Department of Primary Care and Social Medicine, Charing Cross Hospital Imperial College, London, UK ${ }^{4}$ Division of Clinical Psychology, Lund University, Lund, Sweden

\section{Correspondence to} Dr Walter Osika, Stress Research Institute, Stockholm University, SE-106 91

Stockholm, Sweden; walter.osika@stressforskning. su.se

Accepted 1 September 2009 Published Online First 11 October 2009

\section{ABSTRACT \\ Objective Psychosocial adversity is a risk factor for cardiovascular disease (CVD) in adults. The authors assessed associations of reactive hyperaemia peripheral arterial tonometry (RH-PAT), a measure of endothelial function predictive of CVD, with self-assessed psychological health among school children.}

Methods A total of 248 healthy school children (mean (SD) age 14.0 (1.0); 136 girls and 112 boys) underwent RH-PAT testing. They completed the Beck Youth Inventories (BYI) of emotional and social impairment scales, which is used to screen for depression, anxiety, anger and disruptive behaviour.

Results No sex differences were observed for the RH-PAT score. Statistically significant differences were observed for the BYI scores; girls had higher scores for depression, anger and anxiety. Among the girls, there were statistically significant associations between lower RH-PAT scores and higher scores for anger ( $B$ coefficient $=-0.100, p=0.040$ ), depression $(-0.108, p=0.009)$ and anxiety $(-0.138, p=0.039)$ after adjustment for age. Among the boys, disruptive behaviour was associated with higher RH-PAT scores (0.09, $p=0.006$ ).

Conclusions The girls have higher levels of selfassessed anger; depression and anxiety compared with the boys, and these characteristics are associated with lower RH-PAT scores, indicating attenuated endothelial function. Among the boys, disruptive behaviour was associated with better endothelial function. Although psychological ill-health is associated with impaired endothelial function and CVD among adults, such processes may also be relevant to children. Psychosocial adversity in childhood might be a risk factor for subsequent CVD.

Emotional states such as anger, depression, anxiety and psychosocial stress in adults are associated with raised risks of cardiovascular morbidity and mortality ${ }^{1-5}$ but no data exist for such associations among children and adolescents. Psychological factors may be related to early physiological changes such as blood pressure and vascular function in childhood, and this may have a bearing on future risk of cardiovascular disease (CVD).

Depression in the absence of other conventional coronary disease risk factors is associated with abnormal peripheral endothelial function in young adults, ${ }^{6}$ and mental stress induces transient endothelial dysfunction. ${ }^{7}$ It is not known whether an association between early vascular changes such as in the endothelium and self-assessed psychological health exists as early in life as childhood and adolescence.

\section{What is already known on this topic}

- Psychological health is relevant to CVD in adults.

- Acute mental stress and depression is associated with impaired endothelial function in adults.

- Impaired endothelial function is related to increased risk for CVD in adults.

\section{What this study adds}

- Psychological health is associated with endothelial function already in childhood, and the association is more pronounced in girls than in boys.

- Measuring endothelial function in an operator-independent manner is convenient for large-scale field studies.

We wanted to investigate in the young whether endothelial function has an inverse association with increased psychological burden such as increased levels of anger, depression and anxiety, measured as self-rated psychological health.

\section{MATERIALS AND METHODS}

Two schools in the Göteborg region volunteered to participate in this study. In these two schools, 534 healthy school children were invited to participate in the study (mean (SD) age 14.2 (0.9); 12-16 years old; female-male 279:255).

Of these, 248 children (aged 14.0 (1.0), femalemale 136:112) chose to participate and underwent reactive hyperaemia peripheral arterial tonometry (RH-PAT) testing and completed questionnaires. The demographic characteristics of the two schools differed regarding socioeconomic background, with one school with children from a more affluent catchment area and another with children from a more socio-economically disadvantaged area.

Protocols, approved by the ethics committee at Göteborgs University, were presented to the schoolchildren and their parents, and written consent was obtained from both schoolchildren and their parents. 


\section{Study Protocol}

Questionnaires were completed by the children at one time under standard conditions according to previous protocols during school lessons ${ }^{8}$ and put in a coded envelope that was sealed by one of the test leaders. The anthropometric and vascular investigations were performed at the Sahlgrenska University hospital and at a primary healthcare centre by the same person.

Weight was measured using an electronic balance, and height was measured using a stadiometer, allowing calculations of body mass index (BMI, in kilograms per square meter). BMI z scores were calculated according to Cole et al. ${ }^{9}$ Resting blood pressure levels were measured in the right arm with an electronic sphygmomanometer (Welch Allyn, New York, New York, USA). Three separate readings were taken 2 min apart, and the average of the second and third readings was used for analysis. Demographics were assessed as follows: smoking (1 cigarette or more per week), parental education, number of siblings, birth order of the participant, crowding (number of family members per room) and pet in the family.

\section{Reactive hyperaemia: endothelial function}

RH-PAT endothelial function was assessed using the EndoPAT device (Itamar Medical, Caesarea, Israel).

RH-PAT testing is a non-invasive technique that combines traditional flow-mediated dilatation with pneumatic fingertip probes to measure arterial pulse wave amplitude, thus providing a measure of vascular function, which is likely to reflect endothelial function. Fingertip probes are placed on both index fingers, and pulse wave amplitude is recorded for the duration of the study as participants sit in a reclining chair with their hands at heart level. After 5 min of baseline measurements, arterial flow to the arm is occluded for 5 min using a blood pressure cuff inflated to $40 \mathrm{~mm} \mathrm{Hg}$ above the systolic blood pressure (SBP). After the 5 min occlusion, the cuff is rapidly deflated to allow for reactive or flow-mediated hyperaemia. Pulse wave amplitude is recorded for $5 \mathrm{~min}$ after the cuff is deflated. The RH-PAT score is calculated by an integrated software program as the ratio of the average pulse wave amplitude measured over $60 \mathrm{~s}$ starting 1 min after cuff deflation divided by the average pulse wave amplitude measured at baseline. This ratio is normalised to the concurrent signal from the contralateral finger to correct for changes in systemic vascular tone. ${ }^{10}$ In adults, an attenuated RH-PAT score is predictive of coronary heart disease. ${ }^{11} 12$ The technique is operator independent and not subject to subjective interpretations of blood vessel diameter, adjusting for external influence by measuring pulse signals in the contralateral finger. We used the same standard protocol as described in adults. ${ }^{10}$ In a separate group of healthy children $(n=15)$, we investigated the reproducibility of RH-PAT measurements. Each participant was studied twice with a 10 -week interval. The mean value for the first measure of RH-PAT score was 1.862 (range 1.233-3.060); and for the second, 1.818 (1.238-2.762). The mean intrapatient SD of the RH-PAT score was 0.26 , and the coefficient of variation for the RH-PAT score was 14.9. Our results are in line with a previous study where there was a 4 -week interval between the measurements, and the result was very similar to ours, with a mean intrapatient SD of the RH-PAT score being 0.261 and the coefficient of variation for the RH-PAT score being 14.8. ${ }^{13}$

\section{Questionnaires}

The Beck Youth Inventories (BYI) of emotional and social impairment scales were recently introduced as five selfcompletion scales that can be used to assess and screen for depression, anxiety, anger, disruptive behaviour and self-concept in children between the ages of 7 and 18 years. ${ }^{14}$ They have been used to assess psychological health in somatic disease among children in comparison with healthy peers. ${ }^{15} 16$

The depression inventory includes items that reflect children's negative thoughts about themselves and their lives; feeling of sadness and indications of depression. The anxiety inventory includes items that reflect fear and worrying and physiological symptoms associated with anxiety. The anger inventory includes perceptions of mistreatment, negative thoughts about others, feeling of anger and physiological arousal. The disruptive behaviour inventory includes items concerning behaviour and oppositional defiant disorder. The self-concept inventory measures self-perception, and a higher score reflect higher levels of concepts such as social competence, mental strength and positive self-esteem.

Each subscale of 20 items usually takes $<10 \mathrm{~min}$ to complete. The items are written at a second-grade reading level (age approximately 8 years). The participant rates each statement on a four-point Likert scale ranging from never (0) to always (3). The resulting scores are calculated for each inventory by summing their 20 responses, and these scores can range from 0 to 60 .

The psychometric properties of the BYI were studied in a nationally drawn, standardised sample of 800 children in the USA. The internal consistency of all five inventories was high (coefficients $>0.84$ ). ${ }^{17}$ The median 7-day test-retest reliability coefficients for the standardisation sample were computed based on a subsample of 170 individuals aged 7-18 years. These correlations were corrected for the variability of scores on the first testing. Corrected test-retest reliabilities among youth aged 7 through 10 years ranged from 0.74 to 0.90 ; among youth aged 11 through 14 years, from 0.84 to 0.93 ; and among adolescents between the age of 15 and 18 years, from 0.83 to 0.93 .

The inventories have been translated into Swedish, and this version was studied extensively among standardised samples drawn from different Swedish regions and from various clinical and school settings, producing normative data. ${ }^{18}$

\section{Parental education}

The level of parental education was assessed by asking the school children about the highest level of education their parents had achieved. A classification was constructed as used by Statistics Sweden ${ }^{19}$ with a score of 1 for 9-year compulsory school for children aged 7-16 years; 2, for completed upper secondary education; and 3, for university or college or higher education, with separate variables for mothers and fathers. A separate category was identified where a parent was not present. We performed an analysis to confirm that single parenthood was not a confounding factor for associations with this measure of parental education.

\section{Statistical analysis}

Because the distributions of the BYI subscales were skewed, we used the $\operatorname{lnskew} 0$ transformation. Lnskew 0 is a procedure provided by the software package Stata, which transforms the data to minimise skewness by developing a function including logarithmic transformation with addition of a constant. The constants added were 4.323, 5.126, 9.250, 1.402 and 78.4133 for depression, anger, anxiety, disruptive behaviour and self concept, respectively. In all of these measures, skewness was then reduced to $< \pm 0.001$. The RH-PAT score was log transformed before analysis because of its skewed distribution. Unpaired 
t tests for age, weight, height, BMI z score, SBP, diastolic blood pressure (DBP), RH-PAT and the questionnaire scores were performed to assess variation by sex.

Log-transformed RH-PAT score was the dependent variable in multiple linear regressions to examine its association with age, sex, BMI z score and the five log BYI subscales. The analyses were adjusted for age. Age was modelled in five categories and as a continuous measure; and the continuous measure was chosen because it explained more variance. The analyses were performed for girls and boys separately, and the mean BYI scores are presented in table 1 .

Log-transformed RH-PAT and BMI z scores were modelled as continuous measures, and the dependent variables were modelled in linear regression. The distributions of the five log BYI subscales were divided into quartiles and included in models as series of binary dummy variables to avoid assumptions about linearity. However, the best fit was obtained for models using continuous measures, so these were used.

Interaction testing estimated the difference in association between the sexes by creating an interaction term for the stratifying variable (sex) with the characteristic (psychological health) and examining the association of the interaction term with the dependent variable (RH-PAT). ${ }^{20} 21$ Thus, the analysis examined the association of psychosocial factors with RH-PAT separately for both sexes through stratification, whereas the interaction testing assessed the effect modification among the entire sample. Measures of maternal and paternal education level were included in the models as two sets of binary dummy variables. Measures of smoking status, number of siblings, birth order of the participant and pet ownership were included in models as series of binary dummy variables, whereas crowding (number of family members per room) was modelled as a continuous measure.

In a linear multiple regression analysis, the associations of family composition (single parenthood or intact parenthood) and parental education with RH-PAT were analysed.

Statistical significance is indicated as $p<0.05$. We analysed the data using SPSS V.14.0 (SPSS Base V.14.0 User's Guide, New Jersey: Prentice Hall, 2005) and Stata V.9.2 (Stata statistical software Release 9.2, College Station, Texas, USA: Stata, 2006).

\section{RESULTS}

Mean height, weight and SBP were higher in the boys than in the girls, but DBP was lower (table 1). The mean RH-PAT scores were 1.82 with an SD of \pm 0.55 and, after log transformation, $0.56 \pm 0.27$, with no sex differences.

The lnskew0 transformed BYI data are shown in table 1.

Statistically significant differences were seen in the BYI scores, with higher values among the girls than the boys for depression, anger and anxiety. Height and BMI z score were not associated with the RH-PAT score in either sex and, therefore, cannot be confounding factors in any of the analysis performed here.

We studied the associations of RH-PAT with the BYI scores in the boys and the girls separately. In the girls, we found statistically significant associations between lower RH-PAT scores and higher scores for anger, depression and anxiety but not for disruptive behaviour and self-concept (table 2). In the boys, there was a statistically significant positive association between the RH-PAT score and the disruptive behaviour scale (table 3).

Among the boys, the association between disruptive behaviour and a higher RH-PAT was 0.088 (0.023-0.153) when adjusting for both age and parental education. Single parenthood was not a confounding factor.

After adjustment for both age and parental education among the girls, the association between RH-PAT and anger was -0.089 ( $95 \%$ confidence interval (CI) -0.187 to 0.010 ), indicating that parental education was a confounding factor for the association. For depression, the association with RH-PAT was $-0.096(-0.180$ to -0.012$)$; and for anxiety, $-0.123(-0.256$ to $0.009)$.

The association for the anger subscale with RH-PAT had opposite directions for the girls and the boys; in girls, the association was negative but positive in boys (table 3 ). We assessed whether sex was an effect modifier for the association of psychological factors with RH-PAT. The B coefficient for the association of the interaction term for anger with RH-PAT is $-0.188(-0.315$ to -0.061$)$ and for disruptive behaviour $-0.135(-0.220$ to -0.050$)$, indicating statistically

Table 1 Antropometric, physiological and psychological measures in adolescent participants

\begin{tabular}{lccc}
\hline & Females $(\mathbf{n}=\mathbf{1 3 6})$ & Males $(\mathbf{n}=\mathbf{1 1 2})$ & $\mathbf{p}$ Values \\
\hline Age (years) & $14.0(1.0)$ & $14.0(1.0)$ & 0.931 \\
& {$[12.1-16.0]$} & {$[11.9-16.1]$} & \\
Height (m) & $1.64(0.06)$ & $1.71(0.09)$ & $<0.000$ \\
Weight (kg) & $57.1(10.0)$ & $61.9(15.2)$ & 0.005 \\
BMI z score & $0.48(1.09)$ & $0.48(1.11)$ & 1.000 \\
& {$[-2.37$ to 2.87$]$} & {$[-1.86$ to 3.55$]$} & \\
SBP (mm Hg) & $108(8)$ & $110(9)$ & 0.014 \\
DBP (mm Hg) & $60(5)$ & $58(6)$ & $<0.000$ \\
logRH-PAT & $0.57(0.26)$ & $0.55(0.28)$ & 0.574 \\
Depression* & $2.64(0.55)$ & $2.30(0.58)$ & $<0.000$ \\
Anger* & $2.76(0.47)$ & $2.44(0.58)$ & $<0.000$ \\
Anxiety* & $3.10(0.35)$ & $2.82(0.37)$ & $<0.000$ \\
Disruptive behaviour* & $1.68(0.77)$ & $1.70(0.85)$ & 0.883 \\
Self-concept* & $3.54(0.31)$ & $3.47(0.32)$ & 0.099 \\
\hline
\end{tabular}

$\mathrm{p}$ Values between sexes based on independent $\mathrm{t}$ tests. Numbers in square brackets represents range.

${ }^{*}$ As the distributions of the BYI subscales were skewed, we used the Inskew0 transformation procedure provided by Stata to eliminate skewness.

Table 2 The association between RH-PAT and the Beck Youth Inventories in girls

\begin{tabular}{|c|c|c|c|c|c|c|}
\hline & \multicolumn{3}{|c|}{ Unadjusted } & \multicolumn{3}{|c|}{ Adjusted for age } \\
\hline & B & $95 \% \mathrm{Cl}$ & p Value & $\bar{B}$ & $95 \% \mathrm{Cl}$ & p Value \\
\hline Depression & -0.108 & -0.189 to -0.027 & 0.010 & -0.108 & -0.189 to -0.027 & 0.009 \\
\hline Anxiety & -0.130 & -0.260 to 0.000 & 0.050 & -0.138 & -0.268 to -0.007 & 0.039 \\
\hline Disruptive behaviour & -0.047 & -0.105 to 0.012 & 0.118 & -0.042 & -0.101 to 0.017 & 0.162 \\
\hline Self concept & -0.058 & -0.208 to 0.091 & 0.442 & -0.066 & -0.216 to 0.084 & 0.386 \\
\hline
\end{tabular}

The negative coefficient indicates an inverse association of the psychological measure with the dependent measure RH-PAT; that is, a higher depression score is associated with a lower RH-PAT value. 
Table 3 The association between the RH-PAT score and the Beck Youth Inventories in boys

\begin{tabular}{|c|c|c|c|c|c|c|}
\hline & \multicolumn{3}{|c|}{ Unadjusted } & \multicolumn{3}{|c|}{ Adjusted for age } \\
\hline & $\bar{B}$ & $95 \% \mathrm{Cl}$ & p Values & B & $95 \% \mathrm{CI}$ & p Values \\
\hline Depression & 0.005 & -0.089 to 0.098 & 0.923 & -0.003 & -0.098 to 0.092 & 0.956 \\
\hline Anxiety & -0.042 & -0.183 to 0.099 & 0.559 & -0.043 & -0.189 to 0.103 & 0.564 \\
\hline Disruptive behaviour & 0.090 & 0.027 to 0.153 & 0.006 & 0.090 & 0.027 to 0.154 & 0.006 \\
\hline Self concept & 0.019 & -0.151 to 0.189 & 0.824 & 0.020 & -0.151 to 0.191 & 0.817 \\
\hline
\end{tabular}

A positive coefficient indicates an association with the psychological measure with the dependent measure RH-PAT; that is, a higher disruptive behaviour score is associated with a higher RH-PAT value.

significant effect modification. No statistically significant interaction effects were seen by sex for anxiety, depression or self-concept.

Only 15 participants (10 girls; age range $13.7-16.2$ years; mean (SD) $15.3(0.7)$ ) reported that they were smoking 1 cigarette or more per week. When entering the smoking status into regression analysis, it did not alter the associations between self-reported psychological health and RH-PAT. The number of siblings, the birth order of the participant, crowding and pet ownership did not alter the associations between self-reported psychological health and RH-PAT.

There were no significant differences between the schools regarding the RH-PAT or psychometric measures, and the participation rate was equal in the two schools. We included in the model a dummy variable for school but that did not change the associations.

When comparing the 286 participants (female-male 143:143; age 14.3 (0.9)) who did not participate in the study with the 248 participants (female-male 136:112; age 14.0 (1.0); $\mathrm{p}<0.001)$ who underwent RH-PAT testing and completed the questionnaires, there were no significant differences regarding sex between the groups, but the group who participated was younger.

\section{DISCUSSION}

This study shows that self-assessed psychological health, defined as higher levels of anger, depression and anxiety, is related to lower RH-PAT scores (attenuated endothelial function) in girls between 12 and 16 years of age. In the boys, there is an association between disruptive behaviour and higher RH-PAT scores.

We have established a link between psychological health and vascular function in the young and that notable sex differences exists.

In the adults, the RH-PAT score has shown an excellent correlation with measures of coronary and peripheral endothelial dysfunction. ${ }^{10} 11$ Although the RH-PAT technique has been used in children with type 1 diabetes mellitus, to our knowledge, no normative data generated by larger groups of healthy children have been published..$^{13} 22$ The RH-PAT score in the present study was similar for the boys and the girls. Endothelial function in adults is shown to be better in females, ${ }^{23}$ but no sex differences have been reported in children. ${ }^{24}$

The prospective association between anger and coronary heart disease was described initially by investigators from the Framingham Heart Study, who reported that suppressed anger predicted the incidence of coronary heart disease among both men and women ${ }^{25}$ and anger proneness in adults predicted coronary heart disease risk. ${ }^{2}$ This concept is further supported by Räikkönen et al, ${ }^{26}$ who showed that trait anger predicts intima media thickness progression in middle-aged women over 3 years. In a study by Shimbo et al, ${ }^{27}$ an angerprovoking interview impaired both endothelium-dependent and endothelium-independent vasodilation.

In the present study, parental education may help to explain the association of anger and anxiety with RH-PAT scores in the girls but not in the boys. Optimism has been shown to mediate the association between parental education and perceived stress among teens. ${ }^{28}$ Several studies have shown the life-long accumulation of CVD risk with socioeconomic disadvantage. ${ }^{29-31}$

Clearly, childhood conditions and experiences of various kinds are of importance in predicting adult health. Psychosocial factors prevailing in childhood are associated with well-being in adults, and a dose-response relationship between adverse experiences in childhood and adult ischaemic heart disease has been described. ${ }^{32}$

Childhood temperament rated by mothers has been associated with adulthood risk factors such as smoking and educational level and with increased intima media thickness in women. ${ }^{33}$

Our study was not designed as prognostic, and it may well be that the observed link between psychological health and vascular function will disappear when participants are older. However, both observational and longitudinal studies indicate that cardiovascular risk factor such as raised blood pressure among adolescents tracks into adulthood, possibly as a result of increased sympathetic activity. ${ }^{3435}$

The biological mechanisms by which psychological factors influence CVD risk need to be unravelled. Heightened sympathetic arousal and excessive circulating catecholamines are known to cause direct damage to the endothelium, ${ }^{36}$ and impairs endothelium-dependent flow-mediated dilation in healthy volunteers. ${ }^{37}$ This is consistent with our results, as higher levels of anger and anxiety in girls is associated with attenuated endothelial function. In adults, the association of vascular function with mental stress and depression has been demonstrated. ${ }^{67}$ Depressive mood has been linked with prolonged noradrenaline responses to stress $^{38}$ and to abnormalities in the hypothalamic pituitary axis, ${ }^{8}$ which may influence endothelial function. Stress-related endothelial dysfunction can be prevented by blocking cortisol production with metyrapone, demonstrating a direct or facilitative role for cortisol in the development of endothelial dysfunction. ${ }^{39}$

The use of young schoolchildren without known risk factors of ischaemic heart disease makes it likely that psychological health itself, such as self-assessed levels of anger, depression and anxiety and higher difficulty scores, explains the lower RH-PAT scores in girls.

In the present study, the girls had significantly higher scores for anger, depression and anxiety than boys, which is consistent with data from Swedish studies. 1840 


\section{Potential limitations}

While similar measures of endothelial function predict cardiovascular events, the RH-PAT score has not yet been used to evaluate the risk of future cardiovascular events in either adult or paediatric populations. However, reactive hyperaemia, measured in the digital vasculature, has shown relations to multiple traditional and metabolic cardiovascular risk factors. ${ }^{41}$

As the participation rate was relatively low, in particular for the boys, and the participating group was younger compared with the non-participating group, selection bias could have influenced our results, which is important when interpreting the results. One possibility is that more deviant boys chose not to participate in the study, possibly reducing the magnitude of associations among boys. Precise pubertal status was not reported. Although most of the participants were in Tanner stage 4-5, pubertal status could be a confounder, which may influence the sex differences in the associations reported. However, we did adjust for age and that should somewhat attenuate any effect of pubertal status.

In summary, we extend previous findings in adults showing that the association of psychological adversity and illhealth with impaired endothelial function is also valid in the young.

Lower RH-PAT scores, indicating impaired endothelial function, are seen in schoolgirls with increased scores for anger, depression and anxiety. Higher RH-PAT scores were observed among schoolboys with increased scores for disruptive behaviour.

Although our measures in this study did not diagnose either CVD or psychiatric disease, the associations between this early predictive marker for CVD (RH-PAT) and psychological health indicate that psychosocial risks should be considered, particularly in a life-course context. This emphasises the potential importance of early prevention, such as measures to diminish psychological risk among schoolchildren.

Acknowledgements The authors thank the schoolchildren and their parents, teachers and principals at the International School of Gothenburg Region and Utmarksskolan for providing excellent help.

Funding Funding for this study was provided by the Swedish Medical Research Council, The Göteborg Medical Association, The Majblomman Association, Ågrenska Foundation, Sahlgrenska University Hospital, Örebro University Hospital and The Committee for Public Health in the Västra Götaland region.

Ethics approval This study was conducted with the approval of the ethics committee at Sahlgrenska Academy at Göteborgs University, Sweden.

Provenance and peer review Not commissioned; externally peer reviewed.

\section{REFERENCES}

1. Rozanski A, Blumenthal JA, Kaplan J. Impact of psychological factors on the pathogenesis of cardiovascular disease and implications for therapy. Circulation 1999:99:2192-217.

2. Williams JE, Paton CC, Siegler IC, et al. Anger proneness predicts coronary heart disease risk: prospective analysis from the atherosclerosis risk in communities (ARIC) study. Circulation 2000;101:2034-9.

3. Hemingway $\mathbf{H}$, Malik M, Marmot M. Social and psychosocial influences on sudden cardiac death, ventricular arrhythmia and cardiac autonomic function. Eur Heart J 2001;22:1082-101.

4. Rosengren A, Hawken S, Ounpuu S, et al.; INTERHEART investigators. Association of psychosocial risk factors with risk of acute myocardial infarction in 11119 cases and 13648 controls from 52 countries (the INTERHEART study): case-control study. Lancet 2004;364:953-62.

5. Brotman DJ, Golden SH, Wittstein IS. The cardiovascular toll of stress. Lancet 2007;370:1089-100

6. Rajagopalan S, Brook R, Rubenfire M, et al. Abnormal brachial artery flowmediated vasodilation in young adults with major depression. Am J Cardiol 2001;88:196-8, A7.
7. Ghiadoni L, Donald AE, Cropley M, et al. Mental stress induces transient endothelial dysfunction in humans. Circulation 2000;102:2473-8.

8. Osika W, Friberg P, Wahrborg P. A new short self-rating questionnaire to assess stress in children. Int J Behav Med 2007;14:108-17.

9. Cole TJ, Bellizzi MC, Flegal KM, et al. Establishing a standard definition for child overweight and obesity worldwide: international survey. BMJ 2000;320:1240-3.

10. Bonetti PO, Lerman LO, Lerman A. Endothelial dysfunction: a marker of atherosclerotic risk. Arterioscler Thromb Vasc Biol 2003;23:168-75.

11. Bonetti PO, Pumper GM, Higano ST, et al. Noninvasive identification of patients with early coronary atherosclerosis by assessment of digital reactive hyperemia. J Am Coll Cardiol 2004;44:2137-41.

12. Mahmud FH, Earing MG, Lee RA, et al. Altered endothelial function in asymptomatic male adolescents with type 1 diabetes. Congenit Heart Dis 2006;1:98-103.

13. Haller MJ, Stein J, Shuster J, et al. Peripheral artery tonometry demonstrates altered endothelial function in children with type 1 diabetes. Pediatr Diabetes 2007;8:193-8

14. Beck JS, Beck AT, Jolly J. Manual for the Beck Youth Inventories of emotional and social impairment. San Antonio (TX): The Psychological Corporation, 2001.

15. Atwine B, Cantor-Graae E, Bajunirwe F. Psychological distress among AIDS orphans in rural Uganda. Soc Sci Med 2005;61:555-64.

16. Bregnballe V, Thastum M, Schiøtz PO. Psychosocial problems in children with cystic fibrosis. Acta Paediatr 2007;96:58-61.

17. Steer RA, Kumar G, Beck JS, et al. Evidence for the construct validities of the Beck Youth Inventories with child psychiatric outpatients. Psychol Rep 2001;89:559-65.

18. Tideman E. Manual of the Swedish version of the beck youth inventories Stockholm: The Psychological Corporation, 2004.

19. Statistics Sweden 2004. http://www.scb.se/templates/tableOrChart80957.asp (accessed 30 June 2009)

20. Bland JM. An introduction to medical statistics. 3rd edn. Oxford: Oxford University Press, 2000:313-14.

21. Osika W, Ehlin A, Montgomery SM. Does height modify the risk of angina associated with economic adversity? Econ Hum Biol 2006;4:398-411.

22. Haller MJ, Silverstein JH, Shuster JJ. Correlation between radial artery tonometry- and fingertip tonometry-derived augmentation index in children with type 1 diabetes. Diab Vasc Dis Res 2007;4:66.

23. Levenson J, Pessana F, Gariepy J, et al. Gender differences in wall shearmediated brachial artery vasoconstriction and vasodilation. J Am Coll Cardiol 2001;38:1668-74.

24. Järvisalo MJ, Rönnemaa T, Volanen I, et al. Brachial artery dilatation responses in healthy children and adolescents. Am J Physiol Heart Circ Physiol 2002;282:H87-92.

25. Haynes SG, Feinleib M, Kannel WB. The relationship of psychosocial factors to coronary heart disease in the Framingham Study III. Eight-year incidence of coronary heart disease. Am J Epidemiol 1980;111:37-58.

26. Räikkönen K, Matthews KA, Sutton-Tyrrell K, et al. Trait anger and the metabolic syndrome predict progression of carotid atherosclerosis in healthy middle-aged women. Psychosom Med 2004;66:903-8.

27. Shimbo D, Chaplin W, Akinola 0, et al. Effect of anger provocation on endotheliumdependent and -independent vasodilation. Am J Cardiol 2007;99:860-3.

28. Finkelstein DM, Kubzansky LD, Capitman J, et al. Socioeconomic differences in adolescent stress: the role of psychological resources. J Adolesc Health 2007:40:127-34.

29. Kivimäki M, Smith GD, Juonala M, et al. Socioeconomic position in childhood and adult cardiovascular risk factors, vascular structure, and function: cardiovascular risk in young Finns study. Heart 2006;92:474-80.

30. Smith GD, Ben-Shlomo Y, Lynch J. Life course approaches to inequalities in coronary heart disease risk. In: Stansfeld SA, Marmot MG, eds. Stress and the heart: psychosocial pathways to coronary heart disease. London: BMJ Books, 2002:21-49.

31. Pollitt RA, Rose KM, Kaufman JS. Evaluating the evidence for models of life course socioeconomic factors and cardiovascular outcomes: a systematic review. BMC Public Health 2005;5:7.

32. Dong M, Giles WH, Felitti VJ, et al. Insights into causal pathways for ischemic heart disease: adverse childhood experiences study. Circulation 2004:110:1761-6.

33. Keltikangas-Järvinen L, Pulkki-Råback L, Puttonen S, et al. Childhood hyperactivity as a predictor of carotid artery intima media thickness over a period of 21 years: the cardiovascular risk in young Finns study. Psychosom Med 2006;68:509-16

34. Bao W, Threefoot SA, Srinivasan SR, et al. Essential hypertension predicted by tracking of elevated blood pressure from childhood to adulthood: the Bogalusa Heart Study. Am J Hypertens 1995;8:657-65.

35. Chen W, Bao W, Begum S, et al. Age-related patterns of the clustering of cardiovascular risk variables of syndrome $X$ from childhood to young adulthood 
in a population made up of black and white subjects: the Bogalusa Heart Study. Diabetes 2000;49:1042-8.

36. Haft JI. Cardiovascular injury induced by sympathetic catecholamines. Prog Cardiovasc Dis 1974;17:73-86.

37. Hijmering ML, Stroes ES, Olijhoek J, et al. Sympathetic activation markedly reduces endothelium-dependent, flow-mediated vasodilation. J Am Coll Cardiol 2002;39:683-8.

38. Gold SM, Zakowski SG, Valdimarsdottir HB, et al. Higher Beck depression scores predict delayed epinephrine recovery after acute psychological stress independent of baseline levels of stress and mood. Biol Psychol 2004;67:261-73.
39. Broadley AJ, Korszun A, Abdelaal E, et al. Inhibition of cortisol production with metyrapone prevents mental stress-induced endothelial dysfunction and baroreflex impairment. J Am Coll Cardiol 2005; 46:344-50

40. Olsson G, von Knorring AL. Beck's Depression Inventory as a screening instrument for adolescent depression in Sweden: gender differences. Acta Psychiatr Scand 1997;95:277-82.

41. Hamburg NM, Keyes MJ, Larson MG, et al. Cross-sectional relations of digital vascular function to cardiovascular risk factors in the Framingham Heart Study. Circulation 2008;117:2467-74. 